\title{
ANALYTIC MODULI SPACES OF SIMPLE (CO)FRAMED SHEAVES
}

\author{
HUBERT FLENNER AND MARTIN LÜBKE
}

\begin{abstract}
Let $X$ be a complex space and $\mathcal{F}$ a coherent $\mathcal{O}_{X}$-module. A $\mathcal{F}$ (co)framed sheaf on $X$ is a pair $(\mathcal{E}, \varphi)$ with a coherent $\mathcal{O}_{X}$-module $\mathcal{E}$ and a morphism of coherent sheaves $\varphi: \mathcal{F} \longrightarrow \mathcal{E}$ (resp. $\varphi: \mathcal{E} \longrightarrow \mathcal{F}$ ). Two such pairs $(\mathcal{E}, \varphi)$ and $\left(\mathcal{E}^{\prime}, \varphi^{\prime}\right)$ are said to be isomorphic if there exists an isomorphism of sheaves $\alpha: \mathcal{E} \longrightarrow \mathcal{E}^{\prime}$ with $\alpha \circ \varphi=\varphi^{\prime}\left(\operatorname{resp} . \varphi^{\prime} \circ \alpha=\varphi\right)$. A pair $(\mathcal{E}, \varphi)$ is called simple if its only automorphism is the identity on $\mathcal{E}$. In this note we prove a representability theorem in a relative framework, which implies in particular that there is a moduli space of simple $\mathcal{F}$-(co)framed sheaves on a given compact complex space $X$.
\end{abstract}

\section{INTRODUCTION}

Let $X$ be a complex space and $\mathcal{F}$ a coherent $\mathcal{O}_{X}$-module. By a $\mathcal{F}$-coframed sheaf on $X$ we mean a pair $(\mathcal{E}, \varphi)$ with

(a) $\mathcal{E}$ is a coherent $\mathcal{O}_{X}$-module,

(b) $\varphi: \mathcal{F} \longrightarrow \mathcal{E}$ is a morphism of coherent sheaves.

(Following [HL1], HL2], a $\mathcal{F}$-framed sheaf is dually a pair $(\mathcal{E}, \varphi)$ with $\mathcal{E}$ as above and a morphism $\varphi: \mathcal{E} \longrightarrow \mathcal{F}$.) Two such pairs $(\mathcal{E}, \varphi)$ and $\left(\mathcal{E}^{\prime}, \varphi^{\prime}\right)$ are said to be isomorphic if there exists an isomorphism of sheaves $\alpha: \mathcal{E} \longrightarrow \mathcal{E}^{\prime}$ with $\alpha \circ \varphi=\varphi^{\prime}$. A pair $(\mathcal{E}, \varphi)$ is called simple if its only automorphism is the identity on $\mathcal{E}$. The purpose of this note is to show that there is a moduli space of simple $\mathcal{F}$-(co)framed sheaves on a given compact complex space $X$.

More generally, we will show the following relative result. Let $X \rightarrow S$ be a proper morphism of complex spaces. By a family of $\mathcal{F}$-coframed sheaves over $S$ (or a $\mathcal{F}$-coframed sheaf on $X / S$ in brief) we mean a $\mathcal{F}$-coframed sheaf $(\mathcal{E}, \varphi)$ on $X$ that is $S$-flat. Such a family will be called simple if its restriction to each fibre $X(s):=f^{-1}(s)$ is simple.

We consider the set-valued functor $P: \mathfrak{A n}_{S} \longrightarrow \mathfrak{s e t s}$ on the category of complex spaces over $S$ such that $P(T)$ (for $T \in \mathfrak{A n}_{S}$ ) is the set of all isomorphism classes of simple $\mathcal{F}_{T}$-coframed sheaves $(\mathcal{E}, \varphi)$ on $X_{T} / T$, where $X_{T}:=X \times_{S} T$ and $\mathcal{F}_{T}$ is the pullback of $\mathcal{F}$ to $X_{T}$. The main result of this paper is

Theorem 1.1. If $X$ is cohomologically flat over $S$ in dimension 0 , then the functor $P$ is representable by a (not necessarily separated) complex space.

Thus, informally speaking there is a (relative) moduli space of $\mathcal{F}$-coframed sheaves on $X / S$. An inspection of the proof shows that the analogous result holds

1991 Mathematics Subject Classification. 32G13, 14D20.

This paper was prepared during a visit of the second author to the University of Bochum which was financed by EAGER - European Algebraic Geometry Research Training Network, contract No. HPRN-CT-2000-00099 (BBW 99.0030). 
for simple $\mathcal{F}$-framed sheaves provided that $\mathcal{F}$ is flat over $S$. The reason that in the case of $\mathcal{F}$-framed sheaves we need this additional assumption is that only in this case the functor $\operatorname{Hom}(\mathcal{E}, \mathcal{F})$ is known to be representable (see 2.3) whereas $\underline{\operatorname{Hom}}(\mathcal{F}, \mathcal{E})$ is representable as soon as $\mathcal{E}$ is $S$-flat.

Our main motivation for studying moduli spaces of $\mathcal{F}$-(co)framed sheaves is the following. In the case that $\mathcal{F}$ and $\mathcal{E}$ are locally free, a pair $(\mathcal{E}, \varphi)$ as above is called a $\mathcal{F}$-(co)framed vector bundle or holomorphic pair. Various types of holomorphic pairs over compact complex manifolds (e.g. the coframed ones with $\mathcal{F}=\mathcal{O}_{X}$ or the framed ones with arbitrary $\mathcal{F}$, see [OT1, OT2]) can be identified with solutions of so-called vortex equations via a Kobayashi-Hitchin type correspondence. On complex surfaces, these solutions can further be identified with solutions of SeibergWitten equations, and moduli spaces $\mathcal{M}^{\text {st }}$ of stable holomorphic pairs (which are open subsets of the moduli spaces $\mathcal{M}^{s}$ of simple ones) can be used to effectively calculate Seiberg-Witten invariants in several cases.

It is important to notice that the set $\mathcal{M}^{s}$ a priori has two analytic structures. One is given by our result and makes it possible to determine $\mathcal{M}^{s}$ using complexanalytic deformation theory. The other one, which is the one relevant in SeibergWitten theory, is given by a gauge theoretical description as in [LL]. But the main result of that paper is in fact that these two structures are indeed the same.

Finally we mention that moduli spaces of stable $\mathcal{F}$-framed sheaves on algebraic manifolds have been constructed in [HL1].

Without a (co)framing, a moduli space of simple bundles was constructed in [KO and in a more general context in [FS]. In this paper we follow closely the method of proof in the latter paper. The main difficulty is to verify the relative representability of the general criterion 4.3 for the functor $P$ in 1.1. For this we will show in Sect. 2 that the functor of endomorphisms of $\mathcal{F}$-coframed sheaves is representable. In Sect. 3 we show the openness of the set of points where a coframed sheaf is simple. After these preparations it will be easy to give in Sect. 4 the proof of 1.1 .

\section{Preparations}

We start with an algebraic lemma.

Lemma 2.1. Let $R$ be a ring, $L, M, N R$-modules, and $\alpha: L \longrightarrow M, \beta: L \longrightarrow N$ $R$-linear maps. If $K:=\operatorname{coker}((\alpha,-\beta): L \longrightarrow M \oplus N)$, then

$$
\mathbb{S}(K) \cong \mathbb{S}(M) \otimes_{\mathbb{S}(L)} \mathbb{S}(N)
$$

where we consider the symmetric algebras $\mathbb{S}(M), \mathbb{S}(N)$ as $\mathbb{S}(L)$-algebras via the maps $\mathbb{S}(\alpha), \mathbb{S}(\beta)$.

Proof. There are canonical maps $M \longrightarrow K$ and $N \longrightarrow K$ given by

$$
m \mapsto \text { residue class of }(m, 0), n \mapsto \text { residue class of }(0, n) .
$$

These maps induce a commutative diagram

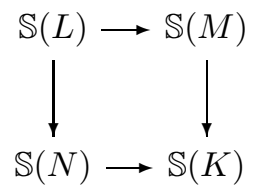


and so, using the universal property of the tensor product, there is a natural map

$$
\mathbb{S}(M) \otimes_{\mathbb{S}(L)} \mathbb{S}(N) \longrightarrow \mathbb{S}(K) .
$$

Conversely, to construct a natural map in the other direction, note that the first graded piece of $\mathbb{S}(M) \otimes_{\mathbb{S}(L)} \mathbb{S}(N)$ is just $(M \oplus N) / L=K$, so there is an induced map

$$
\mathbb{S}(K) \longrightarrow \mathbb{S}(M) \otimes_{\mathbb{S}(L)} \mathbb{S}(N) .
$$

It is easy to check that these maps are inverse to each other.

Now let $S$ be a complex analytic space, and let $\mathfrak{A n}_{S}$ be the category of analytic spaces over $S$. Recall that for a coherent $\mathcal{O}_{S}$-module $\mathcal{F}$ over $S$ the linear fibre space $\mathbb{V}(\mathcal{F})$ represents the functor

$$
F: \mathfrak{A n}_{S} \ni T \mapsto F(T):=\operatorname{Hom}\left(\mathcal{F}_{T}, \mathcal{O}_{T}\right)
$$

(see [Fi] or [EGA, II 1.7]). Note that $F(T)$ has the structure of a $\Gamma\left(T, \mathcal{O}_{T}\right)$-module. Moreover, if $\mathcal{G}$ is another coherent $\mathcal{O}_{S}$-module and $T \mapsto G(T)$ is the associated functor as above, then a transformation of functors $F \longrightarrow G$ will be called linear if $F(T) \longrightarrow G(T)$ is $\Gamma\left(T, \mathcal{O}_{T}\right)$-linear for all $T \in \mathfrak{A n}_{S}$. The reader may easily verify that there is a one-to-one correspondence between such linear transformations of functors and morphisms of sheaves $\mathcal{G} \longrightarrow \mathcal{F}$.

Proposition 2.2. Let $H, F, G: \mathfrak{A n}_{S} \longrightarrow \mathfrak{s e t s}$ be functors that are represented by linear fibre spaces $\mathbb{V}(\mathcal{H}), \mathbb{V}(\mathcal{F}), \mathbb{V}(\mathcal{G})$, respectively. Let $H \longrightarrow G$ and $F \longrightarrow G$ be linear morphisms of functors, and let $K:=H \times_{G} F$ be the fibered product. Then $K$ is represented by $\mathbb{V}(\mathcal{K})$ with

$$
\mathcal{K}:=\operatorname{coker}((\alpha,-\beta): \mathcal{G} \longrightarrow \mathcal{H} \times \mathcal{F}),
$$

where $\alpha: \mathcal{G} \longrightarrow \mathcal{H}$ and $\beta: \mathcal{G} \longrightarrow \mathcal{F}$ are the morphisms of sheaves corresponding to $H \longrightarrow G$ and $F \longrightarrow G$.

Proof. The spaces $\mathbb{V}(\mathcal{H}), \mathbb{V}(\mathcal{F}), \mathbb{V}(\mathcal{G})$ are the analytic spectra associated to the symmetric algebras $\mathbb{S}(\mathcal{H}), \mathbb{S}(\mathcal{F}), \mathbb{V}(\mathcal{G})$, respectively, and $H \times_{G} F$ is represented by $\mathbb{V}(\mathcal{H}) \times_{\mathbb{V}(\mathcal{G})} \mathbb{V}(\mathcal{F})$ which is the analytic spectrum of $\mathbb{S}(\mathcal{H}) \otimes_{\mathbb{S}(\mathcal{G})} \mathbb{S}(\mathcal{F})$. Hence we need to verify that there is a natural isomorphism

$$
\mathbb{S}(\mathcal{H}) \otimes_{\mathbb{S}(\mathcal{G})} \mathbb{S}(\mathcal{F}) \longrightarrow \mathbb{S}(\mathcal{K})
$$

but this is a consequence of Lemma 2.1 .

Let $f: X \longrightarrow S$ be a fixed proper morphism of complex spaces, and let $\mathcal{E}, \mathcal{F}$ be coherent $\mathcal{O}_{X}$-modules, where $\mathcal{E}$ is flat over $S$. Let

$$
H:=\underline{\operatorname{Hom}}(\mathcal{F}, \mathcal{E}): \mathfrak{A n}_{S} \longrightarrow \mathfrak{s e t s}
$$

be the functor given by

$$
H(T):=\operatorname{Hom}_{X_{T}}\left(\mathcal{F}_{T}, \mathcal{E}_{T}\right),
$$

where $X_{T}:=X \times_{S} T$ and $\mathcal{E}_{T}, \mathcal{F}_{T}$ are the pullbacks of $\mathcal{E}, \mathcal{F}$ on $X_{T}$. We recall the following fact.

Theorem 2.3. The functor $H$ is representable by a linear fibre space over $S$. 
For a proof see e.g. [Fl2, 3.2] or [Bi].

Now let $\varphi: \mathcal{F} \longrightarrow \mathcal{E}$ and $\varphi^{\prime}: \mathcal{F} \longrightarrow \mathcal{E}^{\prime}$ be fixed morphisms of coherent sheaves on $X$. Let us consider the functor

$$
M=\underline{\operatorname{Hom}}\left((\mathcal{E}, \varphi),\left(\mathcal{E}^{\prime}, \varphi^{\prime}\right)\right): \mathfrak{A n}_{S} \longrightarrow \mathfrak{s e t s}
$$

defined as follows: For $T \in \mathfrak{A n}_{S}$ the elements of $M(T)$ are the pairs

$$
(c, \alpha) \in \Gamma\left(\mathcal{O}_{X_{T}}\right) \times \operatorname{Hom}_{X_{T}}\left(\mathcal{E}_{T}, \mathcal{E}_{T}^{\prime}\right)
$$

such that the diagram

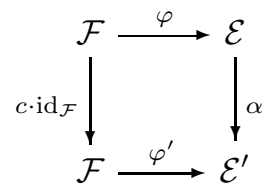

commutes, i.e. such that $c \cdot \varphi^{\prime}=\alpha \cdot \varphi$.

Proposition 2.4. If $\mathcal{O}_{X}, \mathcal{E}$ and $\mathcal{E}^{\prime}$ are flat over $S$, then $M$ is representable by a linear fibre space $\mathbb{V}(\mathcal{M})$ over $S$.

Proof. By Theorem 2.3 the functors

$$
F:=\underline{\operatorname{Hom}}\left(\mathcal{E}, \mathcal{E}^{\prime}\right), H:=\underline{\operatorname{Hom}}\left(\mathcal{O}_{X}, \mathcal{O}_{X}\right), G:=\underline{\operatorname{Hom}}\left(\mathcal{F}, \mathcal{E}^{\prime}\right) \text {. }
$$

are representable by linear fibre spaces over $S$. There are natural maps

$$
H \longrightarrow G, c \mapsto c \cdot \varphi^{\prime},
$$

and

By definition we have

$$
F \longrightarrow G, \alpha \mapsto \alpha \circ \varphi
$$

$$
M=F \times{ }_{G} H,
$$

so the result follows from Proposition 2.2.

An important property of the sheaf $\mathcal{M}$ in Proposition 2.4 is given by

Lemma 2.5. The following are equivalent.

(a) $\mathcal{M}$ is locally free.

(b) For every complex space $T \in \mathfrak{A n}_{S}$ the canonical map

$$
f_{*}\left(\mathcal{H o m}\left((\mathcal{E}, \varphi),\left(\mathcal{E}^{\prime}, \varphi^{\prime}\right)\right)\right) \otimes_{\mathcal{O}_{S}} \mathcal{O}_{T} \longrightarrow f_{T *}\left(\mathcal{H o m}\left(\left(\mathcal{E}_{T}, \varphi_{T}\right),\left(\mathcal{E}_{T}^{\prime}, \varphi_{T}^{\prime}\right)\right)\right)
$$

is an isomorphism.

Moreover, if one of these conditions holds then

$$
\mathcal{M} \cong\left[f_{*}\left(\mathcal{H o m}\left((\mathcal{E}, \varphi),\left(\mathcal{E}^{\prime}, \varphi^{\prime}\right)\right)\right)\right]^{\vee} .
$$

Proof. First note that for every complex space $T \in \mathfrak{A n}_{S}$ we have

$$
\left(\mathcal{M}_{T}\right)^{\vee} \cong f_{T *}\left(\mathcal{H o m}\left(\left(\mathcal{E}_{T}, \varphi_{T}\right),\left(\mathcal{E}_{T}^{\prime}, \varphi_{T}^{\prime}\right)\right)\right) .
$$

Applying this to the case $T=S$, (1) follows immediately from the assumption that $\mathcal{M}$ is locally free. Moreover, if (a) is satisfied we have

$$
\mathcal{M}_{T}^{\vee} \cong \mathcal{M}^{\vee} \otimes_{\mathcal{O}_{S}} \mathcal{O}_{T} \cong f_{*}\left(\mathcal{H o m}\left((\mathcal{E}, \varphi),\left(\mathcal{E}^{\prime}, \varphi^{\prime}\right)\right)\right) \otimes_{\mathcal{O}_{S}} \mathcal{O}_{T},
$$

where for the last isomorphism we have used (1). Thus (b) follows.

Conversely, assume that (b) holds. Using (1) we infer from the isomorphism in (b) that

$$
\mathcal{H o m}\left(\mathcal{M}_{T}, \mathcal{O}_{T}\right) \cong \mathcal{H o m}\left(\mathcal{M}, \mathcal{O}_{S}\right) \otimes_{\mathcal{O}_{S}} \mathcal{O}_{T}
$$


Applying this to $T=\{s\}, s \in S$ a reduced point, it follows that the map

$$
\mathcal{H o m}\left(\mathcal{M}, \mathcal{O}_{S}\right) \longrightarrow \mathcal{H o m}\left(\mathcal{M}, \mathcal{O}_{S} / \mathfrak{m}_{s}\right)
$$

is surjective for every point $s \in S$. Using standard arguments (see e.g. [EGA, 7.5.2]) we conclude that the functor $\mathcal{H o m}(\mathcal{M},-)$ is exact on the category of coherent $\mathcal{O}_{S^{-}}$ modules whence $\mathcal{M}$ is locally free, as required.

\section{Simple $\mathcal{F}$-COfRAmed Sheaves}

As before let $f: X \longrightarrow S$ be a proper morphism of complex spaces, and $\mathcal{F}$ a fixed $\mathcal{O}_{X}$-module. We consider $\mathcal{F}$-coframed sheaves $(\mathcal{E}, \varphi)$ on $X / S$, i.e. $\mathcal{E}$ is a $S$-flat coherent sheaf on $X$ and $\varphi: \mathcal{F} \longrightarrow \mathcal{E}$ is a morphism of $\mathcal{O}_{X}$-modules.

Definition 3.1. $(\mathcal{E}, \varphi)$ is called simple at $s \in S$ if its fibre $(\mathcal{E}(s), \varphi(s))$ is simple, i.e. if

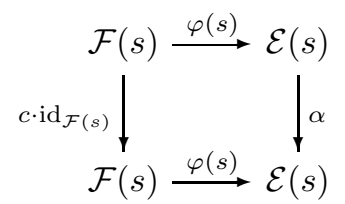

is a commutative diagram, then $\alpha=c \cdot \operatorname{id}_{\mathcal{E}(s)}$. Moreover, $(\mathcal{E}, \varphi)$ is said to be simple over $S$ if it is simple at every point.

Notice that this definition of simpleness of $(\mathcal{E}(s), \varphi(s))$ coincides with the one given in the introduction. Later on we will will need that the points $s \in S$ at which $(\mathcal{E}, \varphi)$ is simple form an open set in $S$. For this we need the following considerations.

By Theorem 2.3 and Proposition 2.4 there are coherent $\mathcal{O}_{S}$-modules $\mathcal{H}$ and $\mathcal{G}$ such that

$$
\underline{\operatorname{End}}(\mathcal{E}, \varphi):=\underline{\operatorname{Hom}}((\mathcal{E}, \varphi),(\mathcal{E}, \varphi)) \text { and } \underline{\operatorname{End}}\left(\mathcal{O}_{X}\right):=\underline{\operatorname{Hom}}\left(\mathcal{O}_{X}, \mathcal{O}_{X}\right)
$$

are represented by $\mathbb{V}(\mathcal{H})$ resp. $\mathbb{V}(\mathcal{G})$. Let

$$
\tilde{a}: \mathcal{G} \longrightarrow \mathcal{H} \text { and } \tilde{b}: \mathcal{H} \longrightarrow \mathcal{G}
$$

be the $\mathcal{O}_{S}$-linear maps associated to the canonical morphisms of functors

$$
\begin{aligned}
& a: \underline{\operatorname{End}}(\mathcal{E}, \varphi) \longrightarrow \underline{\operatorname{End}}\left(\mathcal{O}_{X}\right), \quad \text { resp. } \quad b: \underline{\operatorname{End}}\left(\mathcal{O}_{X}\right) \longrightarrow \underline{\operatorname{End}}(\mathcal{E}, \varphi), \\
& (c, \alpha) \longmapsto c \quad c \longmapsto\left(c, c \cdot \mathrm{id}_{\mathcal{E}}\right)
\end{aligned}
$$

As $a \circ b=\operatorname{id}_{\underline{\text { End }}\left(\mathcal{O}_{X}\right)}$ we have $\tilde{b} \circ \tilde{a}=\operatorname{id}_{\mathcal{G}}$. In other words, $\mathcal{G}$ is a direct summand of $\mathcal{H}$ so that $\mathcal{H} \cong \mathcal{G} \oplus \mathcal{G}^{\prime}$ for some coherent sheaf $\mathcal{G}^{\prime}$ on $S$.

Lemma 3.2. The following are equivalent.

(1) $(\mathcal{E}, \varphi)$ is simple on $S$.

(2) $\mathcal{G}^{\prime}=0$.

(3) The canonical morphism of functors $b: \underline{\operatorname{End}}\left(\mathcal{O}_{X}\right) \longrightarrow \underline{\operatorname{End}}(\mathcal{E}, \varphi)$ is an isomorphism.

Proof. The functor $\underline{\operatorname{End}}(\mathcal{E}(s), \varphi(s))$ resp. $\underline{\operatorname{End}}\left(\mathcal{O}_{X(s)}\right)$ on the category $\mathfrak{A} \mathfrak{n}$ of all analytic spaces is represented by $\mathcal{H}(s)$ resp. $\mathcal{G}(s)$. Thus $(\mathcal{E}(s), \varphi(s))$ is simple if and only if $\mathcal{G}(s) \cong \mathcal{H}(s)$ which is equivalent to the vanishing of $\mathcal{G}^{\prime}(s)$. Using Nakayama's lemma, the equivalence of (1) and (2) follows. Finally, the equivalence of (2) and (3) is immediate from the definition of $\mathcal{G}^{\prime}$. 
Corollary 3.3. If $\mathcal{E}$ is $S$-flat, then the set of points $s \in S$ at which $(\mathcal{E}, \varphi)$ is simple, is an open subset of $S$.

Proof. The set of points $s \in S$ for which $\mathcal{G}^{\prime} / \mathfrak{m}_{s} \cdot \mathcal{G}^{\prime}=0$ is just the complement of the support of $\mathcal{G}^{\prime}$ and hence Zariski-open in $S$. Using Lemma 3.2 we get the desired result.

Recall that a morphism $f: X \longrightarrow S$ of complex spaces is said to be cohomologically flat in dimension 0 if it is flat and if for every $s \in S$ the natural map $f_{*}\left(\mathcal{O}_{X}\right) \longrightarrow f_{*}\left(\mathcal{O}_{X(s)}\right)$ is surjective.

Corollary 3.4. If $(\mathcal{E}, \varphi)$ is simple and $f: X \longrightarrow S$ is cohomologically flat in dimension 0, then $f_{*}\left(\mathcal{O}_{X}\right)$ is a locally free $\mathcal{O}_{S}$-module, and the functor $\underline{\operatorname{End}}(\mathcal{E}, \varphi)$ is represented by $\mathbb{V}\left(f_{*}\left(\mathcal{O}_{X}\right)^{\vee}\right)$.

Proof. The fact that $f_{*}\left(\mathcal{O}_{X}\right)$ is locally free over $S$, is well known (see, e.g. [FS, 9.7]). Moreover, since $(\mathcal{E}, \varphi)$ is simple we have $\underline{\operatorname{End}}(\mathcal{E}, \varphi)=\underline{\operatorname{End}}\left(\mathcal{O}_{X}\right)$. As

$$
\left(f_{T}\right)_{*}\left(\mathcal{O}_{X_{T}}\right) \cong f_{*}\left(\mathcal{O}_{X}\right) \otimes_{\mathcal{O}_{S}} \mathcal{O}_{T}
$$

we get

$$
\operatorname{Hom}_{X_{T}}\left(\mathcal{O}_{X_{T}}, \mathcal{O}_{X_{T}}\right) \cong \Gamma\left(X_{T}, \mathcal{O}_{X_{T}}\right) \cong \operatorname{Hom}_{T}\left(f_{*}\left(\mathcal{O}_{X}\right)^{\vee} \otimes_{\mathcal{O}_{S}} \mathcal{O}_{T}, \mathcal{O}_{T}\right),
$$

so the space $\mathbb{V}\left(f_{*}\left(\mathcal{O}_{X}\right)^{\vee}\right)$ represents $\underline{\operatorname{End}}(\mathcal{E}, \varphi)$ as desired.

\section{Proof of Theorem 1.1}

An isomorphism of two $\mathcal{F}$-coframed sheaves $(\mathcal{E}, \varphi)$ and $\left(\mathcal{E}^{\prime}, \varphi^{\prime}\right)$ is an isomorphism $\alpha: \mathcal{E} \longrightarrow \mathcal{E}^{\prime}$ such that

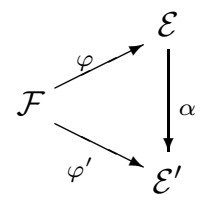

commutes. We note that $(\mathcal{E}, \varphi)$ and $\left(\mathcal{E}^{\prime}, \varphi^{\prime}\right)$ are isomorphic if and only if there is a pair

such that

$$
(c, \alpha) \in \Gamma\left(X, \mathcal{O}_{X}\right) \times \operatorname{Hom}_{X}\left(\mathcal{E}, \mathcal{E}^{\prime}\right)
$$

(a) $c$ is a unit in $f_{*}\left(\mathcal{O}_{X}\right)$,

(b) $\alpha$ is an isomorphism,

(c) $\alpha \circ \varphi=c \cdot \varphi^{\prime}$.

Notice that a simple pair $(\mathcal{E}, \varphi)$ has no automorphism besides id $\mathcal{E}$. If $S$ is a reduced point then the converse also holds, i.e. $(\mathcal{E}, \varphi)$ is simple if and only if id $\mathcal{E}$ is its only automorphism.

Theorem 4.1. Assume that $X$ is cohomologically flat over $S$ in dimension 0 , and let $(\mathcal{E}, \varphi)$ and $\left(\mathcal{E}^{\prime}, \varphi^{\prime}\right)$ be simple pairs. Then the functor

$$
F: \mathfrak{A n}_{S} \longrightarrow \mathfrak{s e t s}, F(T):= \begin{cases}\{1\} & \text { if }(\mathcal{E}, \varphi) \cong\left(\mathcal{E}^{\prime}, \varphi^{\prime}\right), \\ \emptyset & \text { otherwise, }\end{cases}
$$

is representable by a locally closed subspace of $S$. 
Proof. As the sheaf $f_{*}\left(\mathcal{O}_{X}\right)$ is locally free over $S$ we may assume that it has constant rank, say, $r$ over $\mathcal{O}_{S}$. By Proposition 2.4 the functors

$$
M:=\underline{\operatorname{Hom}}\left((\mathcal{E}, \varphi),\left(\mathcal{E}^{\prime}, \varphi^{\prime}\right)\right), M^{\prime}:=\underline{\operatorname{Hom}}\left(\left(\mathcal{E}^{\prime}, \varphi^{\prime}\right),(\mathcal{E}, \varphi)\right)
$$

are representable by linear fibre spaces $\mathbb{V}(\mathcal{M})$ resp. $\mathbb{V}\left(\mathcal{M}^{\prime}\right)$, where $\mathcal{M}$ and $\mathcal{M}^{\prime}$ are coherent $f_{*}\left(\mathcal{O}_{X}\right)$-modules. If for some space $T \in \mathfrak{A n}_{S}$ the pairs $\left(\mathcal{E}_{T}, \varphi_{T}\right)$ and $\left(\mathcal{E}_{T}^{\prime}, \varphi_{T}^{\prime}\right)$ are isomorphic, then by Corollary $3.4 \mathcal{M}_{T}$ and $\mathcal{M}_{T}^{\prime}$ are locally free $\mathcal{O}_{T^{-}}$ modules of rank $r$ on $T$. Thus applying [FS, 9.10] as in the proof of [FS, 9.9], we are reduced to the case that $\mathcal{M}$ and $\mathcal{M}^{\prime}$ are locally free $\mathcal{O}_{S}$-modules of rank $r$. Let us consider the pairings

$$
\begin{aligned}
& M \times M^{\prime} \longrightarrow \underline{\operatorname{End}}(\mathcal{E}, \varphi),((c, \alpha),(d, \beta)) \mapsto(c d, \beta \circ \alpha), \\
& M^{\prime} \times M \longrightarrow \underline{\operatorname{End}}\left(\mathcal{E}^{\prime}, \varphi^{\prime}\right),((d, \beta),(c, \alpha)) \mapsto(c d, \alpha \circ \beta) ;
\end{aligned}
$$

these correspond to pairings

$$
\begin{aligned}
& \mathcal{M}^{\vee} \otimes \mathcal{M}^{\prime \vee} \stackrel{\gamma}{\rightarrow} f_{*}\left(\mathcal{O}_{X}\right), \\
& \mathcal{M}^{\prime \vee} \otimes \mathcal{M}^{\vee} \stackrel{\gamma^{\prime}}{\longrightarrow} f_{*}\left(\mathcal{O}_{X}\right) .
\end{aligned}
$$

Using Lemma 2.5 it follows as in the proof of [FS, 9.9] that our functor $F$ is represented by the open subset

$$
S^{\prime}:=S \backslash \operatorname{supp}\left(\operatorname{coker}(\gamma) \oplus \operatorname{coker}\left(\gamma^{\prime}\right)\right) .
$$

Now we consider the groupoid $\mathfrak{P} \longrightarrow \mathfrak{A} \mathfrak{n}_{S}$, where for $T \in \mathfrak{A n}_{S}$ the objects in

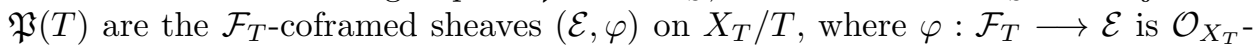
linear. For $(\mathcal{E}, \varphi) \in \mathfrak{P}(T)$ and $\left(\mathcal{E}^{\prime}, \varphi^{\prime}\right) \in \mathfrak{P}\left(T^{\prime}\right)$, a morphism $(\mathcal{E}, \varphi) \longrightarrow\left(\mathcal{E}^{\prime}, \varphi^{\prime}\right)$ is a pair $(f, \alpha)$, where $f: T^{\prime} \longrightarrow T$ is an $S$-morphism and $\alpha: f^{*}(\mathcal{E}) \longrightarrow \mathcal{E}^{\prime}$ is an isomorphism of coherent sheaves such that the diagram

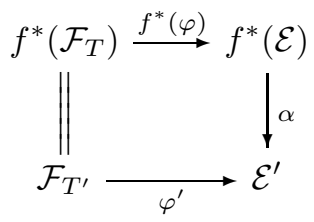

commutes.

\section{Proposition 4.2.}

(a) Every object $\left(\mathcal{E}_{0}, \varphi_{0}\right)$ in $\mathfrak{P}(s), s \in S$, admits a semiuniversal deformation.

(b) Versality is open in $\mathfrak{P}$.

Proof. Let $\mathfrak{Q} \rightarrow \mathfrak{A} \mathfrak{n}_{S}$ be the groupoid where the objects over a space $T \in \mathfrak{A} \mathfrak{n}_{S}$ are the coherent $\mathcal{O}_{X_{T}}$-modules that are $T$-flat. As usual, given $\mathcal{E} \in \mathfrak{Q}(T)$ and $\mathcal{E}^{\prime} \in \mathfrak{Q}\left(T^{\prime}\right)$, a morphism $\mathcal{E} \rightarrow \mathcal{E}^{\prime}$ in $\mathfrak{Q}$ consists of a pair $(f, \alpha)$, where $f: T^{\prime} \rightarrow T$ is an $S$-morphism and $\alpha: f^{*}(\mathcal{E}) \rightarrow \mathcal{E}^{\prime}$ is an isomorphism of coherent sheaves. Assigning to a pair $(\mathcal{E}, \varphi)$ the sheaf $\mathcal{E}$ gives a functor $\mathfrak{P} \rightarrow \mathfrak{Q}$. It is well known that there are semiuniversal deformations in $\mathfrak{Q}$ (see [ST] or [BK]) and that versality is open is $\mathfrak{Q}$ (see e.g., [Fl1]).

The fibre of $\mathfrak{P} \rightarrow \mathfrak{Q}$ over a given object $\mathcal{E} \in \mathfrak{Q}(T)$ is the groupoid $\mathfrak{P}_{\mathcal{E}} \rightarrow \mathfrak{A n}_{T}$ as explained in [Bi, Sect. 10]. More concretely, given a space $Z \in \mathfrak{A n}_{T}$, an object in $\mathfrak{P}_{\mathcal{E}}$ over $Z$ is a morphism

$$
\varphi: \mathcal{F} \otimes_{\mathcal{O}_{S}} \mathcal{O}_{Z} \longrightarrow \mathcal{E} \otimes_{\mathcal{O}_{T}} \mathcal{O}_{Z}
$$


As the functor underlying $\mathfrak{P}_{\mathcal{E}}$ is representable by Theorem 2.3 we get that the objects in $\mathfrak{P}_{\mathcal{E}}(t), t \in T$, admit semiuniversal deformations and that versality is open in $\mathfrak{P}_{\mathcal{E}}$. Applying [Bi, 10.12] gives the desired conclusion.

Before proving the main theorem we remind the reader of the following criterion for the representability of a functor which we present for our purposes in the form as given in [FS, 7.5]; see also [Bi, 3.1] or [KO, §2].

Theorem 4.3. A functor $F: \mathfrak{A n}_{S} \rightarrow \mathfrak{s e t s}$ is representable by a complex space over $S$ (resp. a separated complex space over $S$ ) if and only if the following conditions are satisfied.

(1) (Existence of semiuniversal deformations) Every $a_{0} \in F(s), s \in S$, admits a semiuniversal deformation.

(2) (Sheaf axiom) $F$ is of local nature, i.e. for every complex space $T \in \mathfrak{A n}_{S}$ the presheaf $T \supseteq U \mapsto F(U)$ on $T$ is a sheaf.

(3) (Relative representability) For every $T \in \mathfrak{A n}_{S}$ and $a, b \in F(T)$ the set-valued functor $\operatorname{Equ}(a, b)$ with

$$
\operatorname{Equ}(a, b)(Z):=\left\{\begin{array}{cl}
\{1\} & \text { if } a_{Z}=b_{Z}, \\
\emptyset & \text { otherwise }
\end{array}\right.
$$

is representable by a locally closed (resp. closed) subspace of $T$.

(4) (Openness of versality) For every $T \in \mathfrak{A n}_{S}$ and $a \in F(T)$ the set of points $t \in T$ at which $a$ is formally versal is open in $T$.

Proof of Theorem 1.1. We will verify that the conditions (1)-(4) in Theorem 4.3 are satisfied. (1) and (4) hold by Proposition 4.2. Moreover, (3) is just Theorem 4.1. Finally, (2) holds as simple pairs have no non-trivial automorphism.

\section{REFERENCES}

[Bi] Bingener, J.: Darstellbarkeitskriterien für analytische Funktoren. Ann. Sci. École Norm. Sup. (4) 13, 317-347 (1980).

[BK] Bingener, J.: Lokale Modulräume in der analytischen Geometrie I, II. With the cooperation of S. Kosarew. Aspects of Mathematics D2, D3; Friedr. Vieweg \& Sohn, Braunschweig, 1987.

[Fi] Fischer, G.: Complex analytic geometry. Lecture Notes in Math. Vol. 538. Springer-Verlag, Berlin-New York, 1976.

[Fl1] Flenner, H.: Ein Kriterium für die Offenheit der Versalität. Math. Z. 178, 449-473 (1981).

[Fl2] Flenner, H.: Eine Bemerkung über relative Ext-Garben. Math. Ann. 258, 175-182 (1981).

[FS] Flenner, H.; Sundararaman, D.: Analytic geometry on complex superspaces. Trans. AMS 330, 1-40 (1992).

[EGA] Grothendieck, A.; Dieudonné, J.: Éléments de géométrie algébrique. Publ. Math. IHES 4, 8, 11, 17, 20, 24, 28, 32, 1961-1967.

[HL1] Huybrechts, D.; Lehn, M.: Framed modules and their moduli. Internat. J. Math. 6, 297-324 (1995).

[HL2] Huybrechts, D.; Lehn, M.: The geometry of moduli spaces of sheaves. Aspects of Mathematics, E31. Friedr. Vieweg \& Sohn, Braunschweig, 1997.

[KO] Kosarew, S.; Okonek, C.: Global moduli spaces and simple holomorphic bundles. Publ. Res. Inst. Math. Sci. 25, 1-19 (1989).

[LL] Lübke, M.; Lupascu, P.: Isomorphy of the gauge theoretical and the deformation theoretical moduli space of simple holomorphic pairs. In preparation.

[OT1] Okonek, Ch.; Teleman, A.: The coupled Seiberg-Witten equations, Vortices, and moduli spaces of stable pairs. Internat. J. Math. 6, 893-910 (1995).

[OT2] Okonek, Ch.; Teleman, A.: Gauge theoretical equivariant Gromov-Witten invariants and the full Seiberg-Witten invariants of ruled surfaces. Preprint (2000). 
[Ri] Rim, D.S.: Formal deformation theory. In: Séminaire de Géométrie Algébrique, SGA 7. Lecture Notes in Mathematics 288, Springer Verlag Berlin-Heidelberg-New York 1972.

[ST] Siu, Y.T.; Trautmann, G.: Deformations of coherent analytic sheaves with compact supports. Mem. Amer. Math. Soc. 29 (1981), no. 238.

[Su] Suyama, Y.: The analytic moduli space of simple framed holomorphic pairs. Kyushu J. Math. 50, 65-82 (1996).

Fakultät für Mathematik der Ruhr-Universität, Universitätsstr. 150, Geb. NA 2/72, 44780 Bochum, Germany

E-mail address: Hubert.Flenner@ruhr-uni-bochum.de

Mathematical Institute, Leiden University, PO box 9512, NL-2300 RA Leiden, The NETHERLANDS

E-mail address: lubke@math.leidenuniv.nl 